\title{
LA EXPRESIÓN DE LA PROBABILIDAD Y LA EXPRESIÓN DE LA ORDEN EN ESPAÑOL: DOS FENÓMENOS DE VARIACIÓN SINTÁCTICA
}

\author{
Ana Blanco Canales \\ Universidad de Alcalá \\ ana.blanco@uah.es
}

\begin{abstract}
Resumen
Este estudio tiene como objeto el análisis de la expresión de la posibilidad y la probabilidad y la de la orden y el mandato desde una perspectiva variacionista, esto es, como fenómenos de variación sintáctica en español. Revisaremos, en primer lugar, diferentes planteamientos con respecto a la variación sociosintáctica y las posibilidades de aplicación del modelo laboviano a este nivel lingüístico, hecho que ha sido cuestionado por diferentes autores, dadas las dificultades que entraña encontrar alternancias que no impliquen cambios en el significado referencial ni en la función comunicativa. A continuación, trataremos de explicar los valores semánticos, gramaticales y pragmáticos de estos tipos de expresión desde la teoría de la modalidad lingüística. La última parte se ocupa de mostrar los resultados obtenidos en el análisis cuantitativo y cualitativo de los diferentes recursos para la expresión de la probabilidad y el mandato, análisis que se ha realizado a partir de los datos obtenidos en un corpus de lengua oral. Hemos intentado comprobar si existen o no preferencias por determinadas formas, y si las frecuencias de uso están condicionadas de manera significativa por factores lingüísticos, sociales o estilísticos.
\end{abstract}

PALABRAS CLAVE: modalidad epistémica, modalidad deóntica, variación sintáctica.

\begin{abstract}
The aim of this study is to analyse the expression of possibility and probability and the giving of orders and mandates from a variationist perspective, that is, as cases of syntactic variation in Spanish. We begin by reviewing different approaches to sociosyntactic variation and the scope for applying the Labovian model to this linguistic level, something which some scholars have questioned given the difficulties involved in finding alternatives which do not entail changes in referential meaning or communicative function. We then use the theory of linguistic modality to offer an explanation of the semantic, grammatical and pragmatic values of these types of expression. Finally, we present the results obtained from the quantitative and qualitative analysis of the different resources available for expressing probability and giving orders. This analysis was conducted with data obtained from a corpus of oral language with a view to determining whether or not preferences exist for particular forms and whether the frequency with which they are used is significantly conditioned by linguistic, social or stylistic factors.
\end{abstract}

KEYWORDS: epistemic modality, deontic modality, syntactic variation. 


\section{Sobre la variación sintáctica}

Desde que W. Labov publicó The social stratification of English in New York City (1966) hasta la actualidad, son muchos los trabajos que abordan el análisis lingüístico desde una perspectiva variacionista. No obstante, la mayoría de estos estudios centra su atención en cuestiones fonético-fonológicas, con lo que la variación morfológica, sintáctica, léxica, pragmática, etc. ha quedado relegada a un segundo plano. La causa principal de ello es la dificultad que supone encontrar en niveles no fonológicos alternancias que no impliquen cambios en el significado referencial ni en la función, lo que impediría la aplicación del modelo lavobiano a estos niveles lingüísticos.

Lo que se cuestiona no es la existencia de variación morfosintáctica, que es un hecho indiscutible, sino si las variantes de una variable sintáctica son equivalentes, y en caso de serlo, si alcanzan significación social y/o estilística. Este fue el punto de partida adoptado por B. Lavandera (1984), quien criticó alguno de los resultados obtenidos por defensores de la extensión del modelo sociolingüístico a todos los niveles de análisis, tratando de demostrar que tal variación se debía a condicionamientos lingüísticos y no sociales ni estilísticos ${ }^{1}$. Asimismo, la autora llama la atención sobre un hecho fundamental que en ocasiones se ha malinterpretado: la significación de las frecuencias de las variantes. Según B. Lavandera (1984: 41):

es importante distinguir entre relaciones de frecuencia que son recursos del lenguaje para comunicar información estilística o social, y relaciones de frecuencia que son la consecuencia de la compatibilidad entre los significados referenciales, sociales o estilísticos de algunas formas, y los diferentes contextos en los que pueden aparecer.

B. Lavandera niega toda posibilidad de analogía entre las variantes fonológicas y las no fonológicas, pues considera que nunca dos variantes sintácticas pueden tener el mismo significado, y propone que se sustituya la condición de la identidad referencial por una condición de comparabilidad funcional. Señala, además, la conveniencia de considerar como variables sociolingüísticas solo a aquellas que verdaderamente posean significación social y/o estilística, lo que vendrá determinado por las relaciones entre las frecuencias de sus variantes. En cuanto a la interpretación de los datos, pone de relieve la dificultad que lleva asociada y postula una teoría interpretativa según la cual se podría entender que la covariación entre las variantes y los distintos factores analizados es reflejo de diferencias de estilo comunicativo, en cuyo caso habría que

1 Nos referimos, en concreto, a las objeciones puestas a los resultados que G. Sankoff y S. Labergue lograron en estudios realizados en Montreal. 
determinar cuáles son estos estilos y qué correlaciones relevantes los caracterizan. Solo así se puede llegar a explicar la relación entre comunicación y formas lingüísticas.

Un punto de vista opuesto lo representa, entre otros, D. Sankoff (1992: 187), para quien "las diferencias que afectan al valor referencial o a la función gramatical de las diferentes formas superficiales pueden verse neutralizadas en el discurso". Partiendo de esta premisa, es posible aplicar el método variacionista en todos los niveles de análisis. El autor reconoce que existen diferencias fundamentales entre la variación fonológica y las restantes, pero no cree que esto sea un impedimento para utilizar con estas últimas un método de análisis descriptivo-interpretativo que se apoye en la cuantificación para explicar los datos. Mientras B. Lavandera defiende la necesidad de crear un modelo sociolingüístico único que sirva a todos los niveles de análisis, D. Sankoff entiende este modelo desde una perspectiva más amplia, y asume la necesidad de someterlo a diversas adaptaciones, según el caso. En la variación sintáctica, el investigador no sólo habrá de identificar las variantes, sino que tendrá que determinar cuáles son los contextos en los que se neutralizan. Una vez superados ambos escollos, se puede proceder a un análisis estadístico de características semejantes al que se lleva a cabo con unidades fonológicas. En cuanto a la interpretación de los hechos, señala D. Sankoff (1992: 190) que solo si superamos los estrechos límites de lo puramente sintáctico se puede llegar a la comprensión de los fenómenos estudiados:

podremos tener acceso a los orígenes de la variación sintáctica tan sólo si renunciamos a identificar el conjunto de las variantes posibles con las categorías de un determinado formulismo de la sintaxis autónoma [...]. Para comprender cuál es el origen de este tipo de cambio sintáctico nuestra mirada tiene que proyectarse, en consecuencia, más allá de la sintaxis. Es evidente que durante el proceso de gramaticalización consideraciones propiamente sintácticas pueden resultar cruciales, pero es precisamente en la desdibujada franja que separa el componente sintáctico y el extrasintáctico donde el estudio de la variación sintáctica puede ofrecer sus mayores aportaciones.

Entre ambos puntos de vista, encontramos la postura conciliadora de muchos autores, que proponen diferentes soluciones a la cuestión, relacionadas, en mayor o menor medida, con la pragmática o el análisis del discurso (D. Bolinger, 1977; S. Romaine, 1984; S. Jacobson, 1989). En esta dirección, C. Silva-Corvalán (1988) defiende la posibilidad de extender el análisis variacionista al nivel sintáctico, aunque con restricciones. Señala que no existe similitud entre variación sintáctica y variación fonológica, porque la primera se enfrenta a unas dificultades que no aparecen o que se dan en menor medida en la segunda, como son la escasa cantidad de variación, la dificultad de estudiarla y cuanti- 
ficarla, los problemas que suponen la identificación y definición de los contextos y la cuestión del significado. Pese a todo ello, no descarta la aplicación del modelo variacionista a la sintaxis; considera que sí es posible encontrar variantes sintácticas con el mismo valor referencial, y que ellas deben ser el punto de partida para el análisis. Si se demuestra que estas variantes no llevan asociadas diferencias de significado sintáctico, semántico o pragmático, se puede proceder a su estudio con el mismo método que se aplica a la fonética. Si, por el contrario, se comprueba que las diferencias sobrepasan el nivel lógico o referencial, es entonces cuando podría entenderse la variación como reflejo de estilos de comunicación distintos.

En variación sintáctica es necesario, por tanto, llevar a cabo estudios cualitativos previos que permitan determinar qué condiciones discursivas favorecen la aparición de una variante u otra. Así, si en contextos funcionales iguales un grupo social prefiere unas variantes a las demás, podremos hablar de covariación sociolingüística y tratar estas variantes como integrantes de una variable en sentido estricto. En los últimos años, asistimos a un importante avance de la investigación sociosintáctica, lo que ha sido posible gracias al número de trabajos, pero también a la incorporación de aspectos y procedimientos de otras disciplinas (análisis del discurso, pragmática, etnografía de la comunicación...), que han ampliado las dimensiones y posibilidades de análisis. Se ha producido un aumento considerable de estudios, realizados tanto desde enfoques cuantitativos como aquellos otros de carácter más cualitativo. Los primeros ofrecen como ventaja la exhaustividad del análisis, la fiabilidad que conllevan y la compatibilidad de los resultados. Sin embargo, en ocasiones, el análisis cualitativo puede ser el único camino para el estudio, e incluso, a veces, la información que proporciona resulta más relevante. Por otra parte, el concepto de variante sintáctica ha ido evolucionando, de forma que han quedado atrás las limitaciones y estrecheces que suponían los primeros modelos; las investigaciones se llevan a cabo ahora desde planteamientos multidisciplinares, mucho más flexibles y ricos, y menos apegados a los formalismos metodológicos. Como señala M. J. Serrano (1999: 19), la necesidad de un análisis funcional de la sintaxis ha superado los límites de teorías y corrientes lingüísticas que, en ese esfuerzo por fijar parámetros, podrían obscurecer la interpretación de los hechos del lenguaje. De esta manera, el interés por cuestiones sintáctico-discursivas y sintáctico-pragmáticas ha ido progresivamente en aumento, convirtiéndose en la actualidad en los focos fundamentales de la investigación sintáctica. Los éxitos logrados en ambos niveles animan y aconsejan seguir por este camino (véanse, por ejemplo, los trabajos de C. SilvaCorvalán, 1982, 1984, 2001; P. Martín Butragueño, 1994; P. Bentivoglio 1988, 
1989, 2000; F. Moreno Fernández, 1989; M. J. Serrano, 1994, 1999; L. Cortés Rodríguez, 1991; J. A. Aguado, 1987; E. R. Alcaide, 1990; R. Guillén, 1990; F. Rodríguez-Izquierdo, 1987; C. Sánchez Lanza, 1988; J. Medina López, 1993; C. Fuentes Rodríguez, 1993). La variación sintáctica se estudia actualmente desde una concepción global e integradora de la comunicación, en la que se considera tanto la capacidad referencial como la inferencial del lenguaje, así como el punto de vista del hablante y su intención comunicativa, el contexto determinado en que tiene lugar, además de la necesaria coherencia discursiva.

\section{Dos fenómenos de variación sintáctico-discursiva en español: la expresión} de la probabilidad y la posibilidad y la expresión de la orden y el mandato

\subsection{Sobre el concepto de modalidad}

Cuando hablamos de expresión de la probabilidad y posibilidad y de expresión de la orden y mandato nos encontramos dentro de un área algo difusa de la lingüística, que es el de la modalidad, esto es, estamos hablando de la posición del hablante con respecto a la verdad del contenido de la proposición o a la actitud de los participantes en el acto de la enunciación (E. Ridruejo, 1999: 3211).

La modalidad ha sido estudiada desde diferentes disciplinas (la lógica, la semántica, la psicología, la sintaxis, la pragmática, la teoría de la enunciación), por lo que, en función del autor y del planteamiento, puede remitir a realidades y conceptos muy heterogéneos, como son las modalidades lógicas, las modalidades apreciativas, las modalidades de frase, las modalidades deónticas (obligación), modalidades epistémicas (probabilidad), etc. (T. Otaola, 1988: 98). Desde el punto de vista gramatical, los autores que más han tratado el concepto de modalidad (G. Galichet, A. Bonnard, F. Brunnot, Ch. Bally) han seguido claramente los planteamientos de la lógica y todos ellos coinciden en concebirla como la actitud del hablante con respecto a la realización del contenido de la proposición. Pero a diferencia de los lógicos, los lingüistas parten de la premisa de que las lenguas no se ajustan necesariamente en sus distinciones internas a las diferencias lógicas, dado que, en una lengua, son numerosas las expresiones susceptibles de reflejar una misma modalidad lógica, al tiempo que una sola estructura lingüística puede ser utilizada para expresar diversos tipos de modalidad (E. Ridruejo, 1999: 3212). Un claro ejemplo de esto son los casos que nos ocupan, como ya veremos.

No existe el mismo acuerdo en lo que respecta a las realidades lingüísticas a las que remite el concepto de modalidad, pues se ha relacionado con modos gramaticales, tiempos, verbos modales, adverbios modales, tipos de oraciones (afirmación, interrogación, mandato), etc. En este sentido, para algunos autores, la 
modalidad se identifica exclusivamente con el modo verbal, mientras que para otros incide en toda la frase o enunciado (modalidad expresiva, la impresiva, etc.). Hay autores que la limitan a los matices del pensamiento y del sentimiento, como son la posibilidad, deseo, etc., o las diversas concepciones que el hablante tiene de lo real, irreal y posible. También encontramos autores que combinan varios criterios.

El Esbozo de una Nueva Gramática de la Lengua Española (1978) trata la modalidad oracional distinguiendo en ella el dictum o contenido representativo, esto es, lo que se dice en cada oración, del modus, la manera de decir o actitud subjetiva del hablante respecto a ese contenido. Se identifica, por tanto, el contenido representativo de la oración con el contenido representativo del verbo. La modalidad aparece en el Esbozo como una categoría semántica que tiene que ver con el contenido proposicional, de manera que la oposición realidadirrealidad es la que marcan los modos indicativo y subjuntivo.

Dentro de la tradición europea de análisis lingüístico, se ha propuesto establecer una doble distinción de modalidad, y así, se habla de modalidad del enunciado frente a modalidad de la enunciación (A. Meunier 1974, C. Otaola 1988). La primera se refiere a la manera como el hablante sitúa lo que dice con respecto a la verdad, la falsedad, la probabilidad, la certidumbre, la verosimilitud o en relación con juicios apreciativos, sin que en ello intervengan elementos externos al signo lingüístico. La modalidad de la enunciación corresponde a una relación interpersonal, social y exige una relación entre los protagonistas de la enunciación (D. Maingueneau, 1980), por lo que en este caso, sí intervienen factores del proceso de la comunicación lingüística distintos del enunciado en sí (por ejemplo, en una orden, quedan implicados el hablante y el oyente). Modalidad de la enunciación y modalidad del enunciado son fenómenos diferentes pero compatibles, ya que existe una interacción entre la fuerza elocutiva de los enunciados (la actitud del hablante) y el contenido proposicional que representa.

\subsection{Modalidad epistémica y modalidad deóntica}

La expresión de la probabilidad y de la posibilidad pertenece a lo que se conoce como modalidad epistémica, mientras que la expresión de la orden y el mandato se incluye en la modalidad deóntica. Según E. Ridruejo (1999: 3214), la primera se puede definir como "la expresión del grado de compromiso que el hablante asume con respecto a la verdad de la proposición contenida en un enunciado", mientras que la deóntica supone una formulación de las condiciones en que se establece la verdad del predicado como pertenecientes a un sistema normativo en el que actúa bien el agente o bien el hablante. Aunque 
son modalidades distintas, sin embargo, muestran ciertas coincidencias en tanto, en ambos casos, la proposición a la que se refieren tiene carácter no factual. Por lo que respecta a la expresión de la probabilidad y la posibilidad, el desconocimiento por parte del hablante es manifiesto; en el caso de la expresión del mandato, también existe incertidumbre en tanto el hablante desconoce el cumplimento del contenido de la proposición. Cada uno de los dos tipos de modalidad puede recibir su expresión mediante recursos lingüísticos diversos: la epistémica, a través de adverbios, adjetivos, verbos modales, y también variación en el modo del verbo; la deóntica, a través de la entonación, la sintaxis, o la variación en el modo verbal. Esta variedad de recursos es lo que nos interesa estudiar desde un punto de vista sociolingüístico.

Como señala M. Kitova-Vasilea (2000: 32), la modalidad epistémica constituye una categoría compleja, edificada en dos planos valorativos, el de lo verosímil y el de lo no verosímil, entre los cuales se hallan dos subcategorías modales subjetivas: la de de lo probable y la de lo posible. En principio, es necesario considerarlas por separado, ya que se distinguen tanto desde el punto de vista lógico como lingüístico, aunque entre ellas existen varios puntos en común, dando lugar, de esta forma, a una zona de fluctuación en la que los juicios (más o menos) verosímiles pueden expresarse por medio de estructuras pertenecientes a ambas variedades del estatus epistémico subjetivo. En ellas nos centraremos para nuestro estudio variacionista.

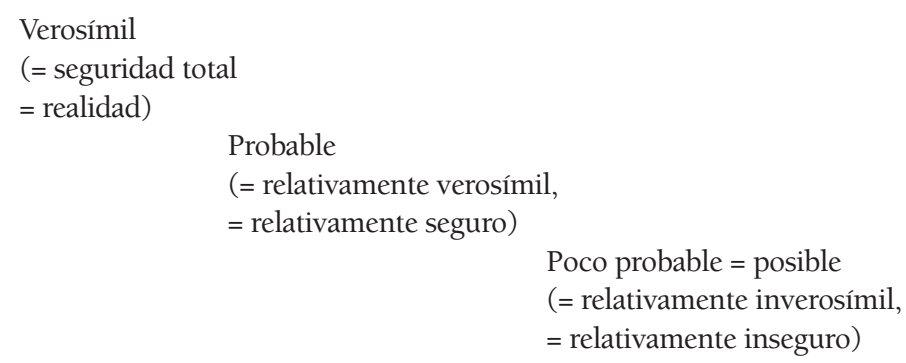

En esta misma línea se expresa S. Nuñez (1991: 161), quien señala que se puede considerar la posibilidad epistémica como una modalidad constituida por dos subcampos modales: el de la probabilidad (deber de) y el de la posibilidad (poder), que se distinguen por las posibilidades de realización de la acción enunciada; esta posibilidad se considera mayor en los límites de la probabilidad epistémica e insignificante en los de la posibilidad. 
Desde un punto de vista semántico, la probabilidad refleja la valoración cuantitativa del nivel de conocimientos del hablante acerca de la realidad, mientras que la posibilidad expresa la sensación subjetiva acerca del grado de veracidad del contenido de lo enunciado por el hablante. En este sentido, señala T. Jiménez Juliá (1989: 203) que la posibilidad se expresa en términos dicotómicos y complementarios: cuando se asegura que algo es posible, se está diciendo al mismo tiempo que es posible que no ocurra, sin que exista posibilidad de graduación. Así, en el enunciado ¿Dónde está Silvia? Quizá esté en el instituto se implica que es posible que esté en el instituto y, al mismo tiempo, que es posible que no esté. La probabilidad, en cambio, se expresa en términos graduales, por lo que podemos decir que algo se considera muy probable o poco probable. En el mismo caso, ¿Dónde está Silvia? Estará en el instituto se implica que es probable que esté en el instituto, pero se excluye como inferencia aceptable que es probable que no esté en el instituto. No obstante, se utilizan en español expresiones como es muy posible o es poco posible, debido a la asociación del término posible al significado de probabilidad, dada la coincidencia de ambos en el valor de incertidumbre.

Por último, hay que señalar que la probabilidad se puede entender como una categoría más abarcadora que la de la posibilidad, dado que todo lo que es probable implica que es posible, pero lo posible, solo a veces es probable. De esta manera podemos entender la estructura epistémica como un continuum dividido en diversos planos: lo verosímil - lo probable o muy probable - lo probable y posible - lo posible - lo inverosímil. En este continuum existe un tramo en el que la expresión de la probabilidad y la posibilidad vienen a confluir, utilizando indistintamente los recursos de una y otra submodalidad.

Por lo que respecta a la modalidad deóntica, cabe señalar la frecuente confusión entre los valores de la 'obligación' y los de la 'necesidad', puesto que ambos conceptos no han quedado bien delimitados en los estudios lingüísticos. Pero, tal y como explica J. Lyons (1980), las obligaciones -morales y legales- y la necesidad física son hechos culturales que dependen de cada comunidad de habla. De esta manera, la modalidad deóntica expresará obligación o necesidad dependiendo de la razón o causa de la obligación, por lo que su valor solo se podrá fijar atendiendo, en cada caso, a factores contextuales y discursivos.

El tratamiento de la modalidad deóntica es, igualmente, inseparable del carácter performativo que la caracteriza, lo que hace imprescindible, una vez más, el análisis pragmático de los diversos factores que confluyen en su interpretación. Su estudio nos sitúa frente a actos como ordenar, rogar, suplicar, aconsejar, pedir, permitir, invitar, advertir, aconsejar, etc., es decir, toda una serie de fuerzas ilocutivas que persiguen la realización de actos controlables 
por parte de un agente, hecho este que supone un conjunto de restricciones semánticas en la predicación. El estudio de los recursos de modalidad deóntica nos lleva, por tanto, al análisis de los enunciados directivos, aquellos que "proponen una determinada actuación o comportamiento indicando que esta debe o puede ser llevada a cabo", sin especificar si el agente de la acción propuesta coincide con el agente (caso de las órdenes), con el hablante (como en las promesas) o con terceros participantes (como en las órdenes indirectas y, en cierta medida, en los enunciados optativos) (S. Núñez, 1991: 137).

No obstante, hay que señalar que, a pesar de que comúnmente es utilizada con valor perfornativo, imponiendo una obligación o concediendo un permiso, este carácter preformativo no define la modalidad deóntica, porque no todos los enunciados deónticos son performativos: el hablante se puede limitar a enunciar que la obligación y el permiso existen. En nuestro estudio nos centraremos solo en aquellos casos claros de influencia sobre el oyente (valor performativo).

Desde el punto de vista de la enunciación (la actitud del hablante con respecto al oyente), la modalidad deóntica se corresponde con lo que se ha denominado modalidad imperativa o exhortativa.

\subsection{Nuestro estudio}

Como ya se ha mencionado, son numerosos los recursos lingüísticos con los que cuenta el español para referirse a aquello de lo que no está seguro o para expresar una orden a su/s interlocutor/es. Así lo podemos ver en los dos esquemas que siguen:

\section{Recursos para la expresión de la posibilidad y la probabilidad ${ }^{2}$}

\section{Expresar lo que se considera probable o muy probable}

1.1. seguramente, seguro que + indicativo o tiempo virtual: expresa información que el hablante cree que va a cumplirse.

1.2. probablemente + indicativo o subjuntivo: refiere hechos a los que se le otorga seguridad pero algo menos que en el caso anterior.

1.3. es probable que + subjuntivo: indica situaciones que el hablante considera que van a realizarse, pero con reservas.

1.4. deber de, tener que + infinitivo: ambas perífrasis indican hechos que se consideran muy probables, cuya realización está dentro de lo previsible y normal.

2 Seguimos la clasificación de F. Matte Bon (1995: 275-263), a la que hemos añadido algunos subapartados y diversas matizaciones. 
1.5. NO + poder + infinitivo: rechaza una probabilidad que acaba de mencionarse explícita o implícitamente en el contexto anterior.

1.6. tiempos virtuales: futuro simple/compuesto; condicional simple/ compuesto.

- futuro simple: probabilidad en el presente.

- futuro compuesto: probabilidad en el pasado del presente (temporalmente, es semejante al pretérito perfecto compuesto).

- condicional simple: probabilidad en el pasado.

- condicional compuesto: probabilidad en un momento anterior a otro momento pasado.

2. Expresar lo que se considera posible

2.1. a lo mejor + indicativo: expresa lo que se considera posible cuando se trata de una hipótesis nueva.

2.2. quizá, tal vez, posiblemente + indicativo o subjuntivo; puede (ser) que + subjuntivo: expresa tanto hipótesis nuevas como las ya evocadas en contextos anteriores.

2.3. es posible que + subjuntivo: refiere hipótesis en cuyo cumplimiento se tienen pocas esperanzas.

\section{Expresar lo que se considera posible pero remoto}

3.1. igual + indicativo: expresa hipótesis que el hablante entiende muy posibles pero a la vez sorprendentes.

3.2. capaz + subjuntivo: indica una idea posible pero remota.

3.3. puede incluso que + subjuntivo: expresa hipótesis lejana.

Recursos para la expresión de la orden y el mandato (influir en el oyente)

1. Imperativo. Dependiendo de la entonación, el tono y volumen de la voz, los gestos y el acompañamiento de partículas, la forma del imperativo puede expresar diferentes grados y matices de influir en el oyente.

1.1. Instrucción: Agite el medicamento antes de usarlo.

1.2. Propuesta: Venga, vámonos al cine.

1.3. Consejo: Descansa un poco, lo necesitas.

1.4. Petición: Pásame la sal.

1.5. Deseo: Diviértete.

1.6. Orden: Sal de mi casa ahora mismo.

2. $a+$ imperativo. Expresa una orden informal.

Venga, todo el mundo a estudiar. 
3. que + subjuntivo. Además de para las órdenes indirectas a terceras personas, esta construcción se emplea para repetir una orden directa ya dada que la otra persona no ha entendido o no ha cumplido (en cuyo, caso resulta descortés).

Que pase el siguiente.

¿Que me dejes en paz de una vez!

\section{Presente de indicativo.}

4.1. Como pregunta, es una forma cortés (pero sin exceso) muy habitual para formular una petición. Si queremos acentuar la cortesía tenemos que anteponer los verbos poder o importarte/le (y a veces querer), en presente o en condicional (muy formal).

¿Me dejas el coche esta tarde, por favor?

4.2. Si no está dentro de una pregunta, se utiliza para dar instrucciones $y$, en relaciones de confianza, para dar órdenes generalmente descorteses, a no ser que cerremos la frase con ¿vale?, ¿de acuerdo? Es muy frecuente la anteposición de diferentes combinaciones de pues, ahora y los verbos ir y coger (muy coloquial) para mostrar enfado ante la actitud negativa del oyente.

Tiras el dado y mueves la ficha tantas casillas como indique el dado.

¿Que no tienes por qué recoger la mesa? Pues ahora vas y te quedas sin salir esta tarde

5. Futuro de indicativo. Forma de orden y mandato fuerte y descortés. Solo se utiliza en situaciones de asimetría social muy marcada.

Tú harás lo que yo te diga.

6. Perífrasis de obligación: deber, tener que

7. Otras formas. Existen determinados enunciados más o menos formularios que cumplen esta misma función en la comunicación.

Ya está bien de ponerte borde.

A ver si animamos el juego.

Muchos de estos recursos, tienen un contexto de uso específico, se utilizan con una intención pragmática determinada y poseen un valor único en la comunicación. Sin embargo, algunos otros son comparables funcionalmente y, por 
ello, intercambiables (el uso de uno u otro no afecta al valor referencial del enunciado) y parecen ser el resultado de estilos propios o particulares (individuales o grupales) de comunicación. En nuestro estudio, nos hemos centrado en estos últimos y hemos querido comprobar si esas preferencias estaban condicionadas o no por factores sociales o estilísticos. Para ello, hemos llevado a cabo un estudio cuantitativo de carácter variacionista a partir de los ejemplos encontrados en nuestro corpus de datos.

El material oral con el que hemos trabajado estaba constituido por grabaciones de habla de registro formal e informal procedentes de 30 hablantes de Alcalá de Henares. Estos 30 individuos formaban una red social cuya características más destacable es el hecho, algo extraordinario en esta localidad, de haber nacido todos en Alcalá con la excepción de dos de ellos, de origen extremeño, que vinieron aquí durante su primer año de vida. De estas 30 personas, 17 eran mujeres y 13 hombres, con edades comprendidas entre los 16 y 84 años. No existía dentro de la red diversidad social relevante, pues todos sus miembros pertenecían a las clases baja y media-baja; sin embargo, sí manifestaba diversidad en cuanto al nivel de instrucción. Así, encontramos desde informantes analfabetos o semi-analfabetos hasta otros con estudios superiores. Para la obtención de los materiales se siguieron dos técnicas: la observación participativa y la entrevista, con lo que se lograron datos de dos registros bien diferenciados (el formal y el informal). En total se analizaron 720 minutos de habla informal. Idéntica cantidad se utilizó de las entrevistas formales: transcribimos los 15 primeros minutos de cada grabación por considerar que en ellos se encontraría una mayor formalidad. Trabajamos, por consiguiente, con 1440 minutos de grabación.

\subsubsection{La expresión de la posibilidad y de la probabilidad}

Siguiendo los criterios mencionados más arriba, hemos elegido para este estudio aquellas formas en las que la diferencia entre lo posible y lo probable se diluyen, esto es, las que expresan al tiempo lo que es probable y posible. Hemos buscado los casos en los que cada enunciado puede expresarse, al menos, con otra de las opciones sin que haya cambios semánticos o pragmáticos. No hemos tratado de conseguir equivalencia absoluta entre las diferentes variables que íbamos a analizar; nos ha resultado suficiente con que cada ejemplo pueda expresarse de otra forma sin que se produzca cambio en el valor referencial. Esto nos ha llevado a establecer cuatro variables; nuestro propósito es analizar las frecuencias de cada una de ellas y de comprobar si existen condicionantes que determinen en mayor o menor medida la presencia de estas formas. Hemos considerado como factores de incidencia los siguientes: 
- naturaleza semántica del verbo:

pensamiento (p)

lengua (1)

percepción (v)

deseo/voluntad (m)

otros (o)

- registro:

formal (f)

informal (i)
- edad:

$1^{a}$ generación (1)

$2^{a}$ generación (2)

$3^{\mathrm{a}}$ generación (3)

$4^{\mathrm{a}}$ generación (4)

- sexo:

hombre (h)

mujer (m)

- nivel de instrucción:

analfabetos o estudios primarios (A)

estudios medios (B)

estudios superiores (C)

Los recursos de posibilidad y probabilidad (en adelante, probabilidad para referirnos tanto a lo probable como a lo posible) y sus correspondientes frecuencias aparecen a continuación.

\begin{tabular}{|l|c|c|}
\hline Probabilidad con subjuntivo + partícula & 46 & $8 \%$ \\
\hline Probabilidad con indicativo + partícula & 224 & $38 \%$ \\
\hline Probabilidad con futuro/condicional & 280 & $48 \%$ \\
\hline Probabilidad con futuro/condicional + partícula & 32 & $5 \%$ \\
\hline & 582 & $100 \%$ \\
\cline { 2 - 3 }
\end{tabular}

Cuadro 1: frecuencias de diferentes recursos para la expresión de la probabilidad

\section{Probabilidad con futuro/condicional}

Se trata de un recurso de gran eficacia si tenemos en cuenta que aúna expresividad, escaso grado de compromiso y simplicidad, pues con solo una unidad lingüística comunicamos un contenido de máxima probabilidad sin "comprometernos" en la valoración de la cantidad de probabilidad, tal y como sucede con las partículas y con la oposición indicativo/subjuntivo.

Las cifras no dejan lugar a dudas sobre el predominio de este recurso para la expresión de la probabilidad en la conversación, predominio que se acentúa si se trata de un verbo de lengua y, en menor medida, si es de pensamiento. Frente a ellos, los verbos de orden y deseo y los de percepción son los más reacios a esta solución. 


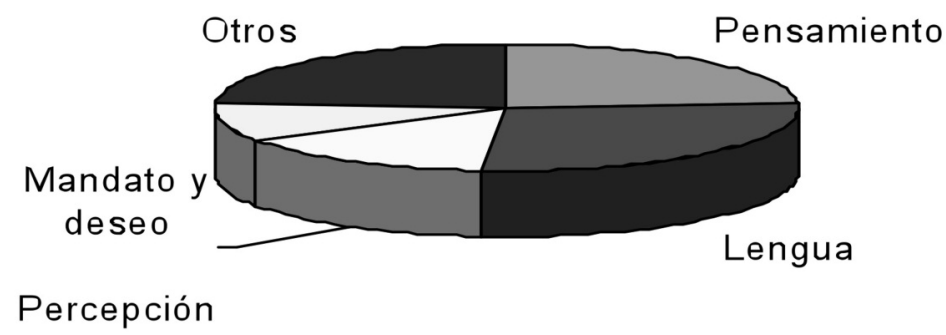

Gráfica 1: distribución del futuro/condicional según la naturaleza semántica del verbo

Si atendemos al reparto por registros vemos que las frecuencias aumentan en el informal (58\% frente a 40\%), el más propenso a modos de enunciación muy expresivos, pero de estructura sencilla. También es mayor su uso entre hombres (43\%) que entre mujeres (54\%), si bien las diferencias no son concluyentes.

El factor generacional muestra que la presencia de este recurso disminuye de forma proporcional a la edad, de manera que son los jóvenes los que se manifiestan más adversos. No obstante, en los cuatro grupos es la forma mayoritaria, por lo que parece lógico pensar que no estamos ante un abandono paulatino de una determinada forma sino ante la progresión de otras, con las que entra en alternancia. Este avance es más perceptible en la población joven.

Contrariamente a la edad, no existe un reparto gradual en función de la instrucción, pues los grupos inferior y superior arrojan cifras similares (52\% y $51 \%$ ), mientras que es el grupo medio el que se aparta de este comportamiento común (37\%).

\section{Probabilidad con indicativo + partícula de duda}

La expresión de la probabilidad a través del indicativo + partículas dubitativas es el segundo recurso más frecuente en nuestros materiales. El primer dato de interés que destaca tras el cálculo de frecuencias por factores es lo determinante que resulta para la elección del modo el tipo de partícula. Así, se observa que a lo mejor exige casi exclusivamente indicativo ${ }^{3}$; no sé acompaña en proporciones semejantes a indicativo y a condicional (55\% / 45\%) $)^{4}$; quizás, tal vez aparece junto a indicativo el $45 \%$ de las ocasiones, porcentaje más alto incluso que con subjuntivo (41\%); posiblemente, probablemente... constituyen la única clase de partículas que se muestra claramente contraria a este modo

3 El uso normativo no tolera a lo mejor + subjuntivo, pero sí más condicional.

4 Por cuestiones puramente prácticas hablamos de indicativo y condicional como modos distintos, sin plantearnos aquí la conveniencia teórica de esta separación. 
(15\%); por último, expresiones como alrededor de, unos, etc. favorecen notablemente el uso del indicativo (75\%).

La naturaleza semántica del verbo ha evidenciado también la preferencia de ciertas clases por unas u otras formas de probabilidad. Comprobamos que los verbos de percepción seguidos de los de mandato y deseo son muy propicios a expresar este contenido en indicativo, mientras que los de pensamiento y lengua optan por el condicional.

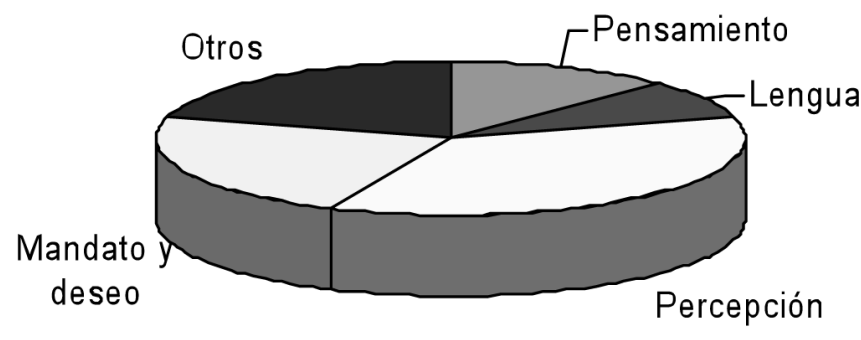

Gráfica 2: distribución del indicativo + partícula según la naturaleza semántica del verbo

Las diferencias por registros son poco significativas (formal - $41 \%$ / informal - 35\%); el sexo, por su parte, presenta mayor contraste (mujeres - 44\% / hombres - 32\%), aunque tampoco es determinante. En cuanto a la edad, destaca sólo la baja cifra que alcanzan los miembros de la cuarta generación (25\%), en clara oposición a las del resto de los grupos, que manifiestan un uso mayor, especialmente, los de la primera generación (40\%, 43\% y 60\%).

Algo semejante ocurre con el parámetro de la instrucción: los niveles A y B (inferior $41 \%$ y medio $49 \%$ ) son favorecedores de la probabilidad en indicativo mientras que el C (superior 24\%) hace uso de este recurso la mitad de las veces que los anteriores.

\section{Probabilidad con subjuntivo + partícula de duda}

Se encuentra en evidente desventaja con respecto a los dos casos anteriores. En su presencia es determinante la partícula que lo acompaña, pues solo quizás, tal vez (41\%) por un lado, y posiblemente, probablemente... (62\%), por otro, admiten -y propician además- el modo subjuntivo; el resto de las partículas consideradas lo rechaza por completo.

El valor semántico del verbo es en esta ocasión poco aclaratorio. El dato más relevante al respecto es que no ha aparecido ningún ejemplo de probabilidad con subjuntivo + partícula en los verbos de percepción. Los que muestran 
mayor facilidad a usarse en este modo son los de pensamiento y mandato y deseo, que superan la media general del $8 \%$ de forma considerable (20\%).

El subjuntivo para la expresión de la probabilidad es ligeramente superior en estilos de habla formales, en los hombres y en hablantes cultos; la edad no incide prácticamente nada.

\begin{tabular}{|l|l|}
\hline hombres & $26-10 \%$ \\
\hline mujeres & $20-6 \%$ \\
\hline
\end{tabular}

Cuadro 3: frecuencias según el sexo

\begin{tabular}{|l|l|}
\hline$A$ & $12-4 \%$ \\
\hline$B$ & $12-9 \%$ \\
\hline C & $22-16 \%$ \\
\hline
\end{tabular}

\begin{tabular}{|l|l|}
\hline formal & $36-11 \%$ \\
\hline informal & $10-4 \%$ \\
\hline
\end{tabular}

Cuadro 5: frecuencias según el registro

\section{Probabilidad con futuro/condicional + partícula de duda}

El porcentaje de aparición de esta fórmula es bajísimo (5\%). Posiblemente este rechazo se explique por la redundancia que supone la confluencia de los dos elementos por excelencia de la probabilidad. Por otra parte, la presencia o ausencia de las partículas de significado dubitativo no modifica el valor del verbo; así, en los siguientes enunciados

(1) Alcalá tendría 10.000 habitantes

(2) Alcalá a lo mejor tendría 10.000 habitantes

a lo mejor es una unidad innecesaria para la comprensión exacta del mensaje. No ocurre esto cuando se trata del indicativo, ya que se producen cambios sustanciales de significado (Alcalá tenía 10.000 habitantes / Alcalá a lo mejor tenía 10.000 habitantes), o del subjuntivo, que exige la aparición obligatoria de dichas partículas.

Un dato importante que hay que destacar en relación a las partículas es que cuando tenemos no sé la frecuencia del futuro/condicional es muy alta (45\%). Otras unidades como probablemente, posiblemente... o como alrededor de, unos, aunque no logran cifras muy elevadas, presentan índices de uso nada desdeñables ( $23 \%$ y $25 \%$ respectivamente).

No aparece nunca futuro/condicional + partícula con verbos de percepción, mandato o deseo. Por lo que respecta al registro, la mayor parte de los casos se acumulan en el formal. Por otra parte, no se aprecian apenas diferencias entre hombres y mujeres. Si nos fijamos en la edad, se percibe muy tímidamente que cuanto más joven es el hablante, mayor uso hace de esta solución. Lo más destacable en este sentido es la ausencia total de ejemplos entre informantes de la cuarta generación. Por último, cabe señalar que los porcentajes son proporcionales al nivel de instrucción (a mayor formación mayor índice porcentual), 
pero se trata de diferencias muy poco relevantes, dada la escasa incidencia de este recurso en la totalidad de los materiales.

De los cuatro recursos para la expresión de la probabilidad dos manifiestan notable vitalidad (futuro/condicional e indicativo + partícula) y otros dos muestran, en general, un uso muy reducido (subjuntivo + partícula y futuro/condicional + partícula). No obstante, el análisis conjunto de formas lingüísticas y factores condicionantes nos describe en algunos casos una situación diferente, pues, como sucede muchas veces, la globalidad de los datos oculta la realidad. Así, el elemento no sé se combina con indicativo y con futuro/condicional en proporciones muy cercanas; lo mismo ocurre con quizás en relación al indicativo y al subjuntivo. Las partículas posiblemente, probablemente... exigen subjuntivo en la mayoría de los casos. Por otra parte, hay verbos, como los de lengua y los de percepción, que favorecen claramente uno de los recursos (futuro/condicional los primeros, indicativo + partícula los segundos), mientras que otros -verbos de pensamiento, mandato y deseo- admiten cierta variedad (los verbos de pensamiento alcanzan el mismo porcentaje con indicativo que con subjuntivo; los de mandato y deseo, en subjuntivo + partícula y en futuro/condicional). El dato más relevante con respecto al parámetro generacional es el predominio absoluto de la solución sin partículas entre los más mayores, grupo en el que, además, no se ha documentado ningún ejemplo de futuro/condicional + partícula; en los grupos restantes, en especial en el primero, se aprecia un reparto algo más equitativo. En cuanto al nivel de instrucción, son los más cultos los que ofrecen un comportamiento más diversificado, lo que unido al hecho anterior, puede interpretarse como leve avance de los recursos en desventaja, y en última instancia, como una tendencia a enriquecer los medios lingüísticos para la expresión de la probabilidad.

\subsubsection{La expresión de la orden y el mandato}

El imperativo es la forma lingüística propia de la orden y el mandato. Pero hay en español otras posibles realizaciones para la expresión de este contenido, entre las que destacan el futuro de indicativo, el presente de indicativo, el infinitivo ${ }^{5}$, que + subjuntivo o las oraciones interrogativas.

Existen enunciados que dentro de un discurso determinado tienen como función la exhortación o la orden, pero son ocasionales y, además, muy difíciles de sistematizar. Algunos ejemplos de este tipo son:

5 Nos referimos al imperativo como tal, no al imperativo que por razones fonéticas se ha confundido en la segunda persona del plural con el infinitivo (a comer, a callarse). 
(3) Bueno, ya está bien de ponerte borde 6 . (inf. 9)

(4) Bueno, a ver si animamos un poquito el juego ${ }^{7}$. (inf. 21)

Por otra parte, algunas perífrasis obligativas (deber, tener que) se utilizan también para dar órdenes, pero resulta complejo discernir cuál de los dos valores (el de obligación o el de necesidad) predomina en cada ocasión. Sólo el contexto y la entonación pueden indicar si deber es un consejo o una orden. En cuanto a tener que, además del contexto, hay que considerar si la obligación la impone el hablante o si viene determinada por otros, pues en el primer caso estaríamos ante una orden mientras que en el segundo se trataría sólo de una información (E. Lorenzo, 1971: 129-130).

Por último, funcionan igualmente como indicadores y reforzadores de mandatos expresiones como hala, venga, vamos, anda.

En nuestros materiales hemos documentado todos estos casos, aunque en porcentajes muy dispares:

\begin{tabular}{|l|c|c|}
\hline Imperativo & 424 & $75,7 \%$ \\
\hline presente & 70 & $12,5 \%$ \\
\hline Futuro & 14 & $2,5 \%$ \\
\hline que + subjuntivo & 36 & $6,4 \%$ \\
\hline oraciones interrogativas & 16 & $2,8 \%$ \\
\hline infinitivo & 2 & $0 \%$ \\
\hline & 560 & $100 \%$ \\
\hline
\end{tabular}

Cuadro 6: frecuencias de las formas para la expresión de la orden y el mandato

Para su análisis se atendió a los siguientes parámetros:

- naturaleza semántica del verbo:

pensamiento (p)

lengua (l)

percepción (v)

deseo/voluntad (m)

otros (o)
- edad:

$1^{a}$ generación (1)

$2^{a}$ generación (2)

$3^{a}$ generación (3)

$4^{\mathrm{a}}$ generación (4)

6 En opinión de E. Lorenzo (1971: 127) ésta es una fórmula de gran expresividad, característica que debe al valor anticipatorio del verbo, que expresa más el resultado que la orden.

7 El operador lingüístico a ver si puede tener, además, valor de expectativa o temor (Bueno, a ver si ha venido ya). 
- relaciones entre los informantes

+ poder (p)

- $\operatorname{poder}(\mathrm{n})$

+ solidaridad (s)

- registro:

formal (f)

informal (i)
- sexo:

hombre (h)

mujer (m)

- nivel de instrucción:

analfabetos o estudios primarios (A)

estudios medios (B)

estudios superiores (C)

\section{El imperativo}

Los datos no dejan lugar a dudas en cuanto al predominio absoluto del imperativo como forma de expresión de la orden. A pesar de la gran cantidad de enunciados cuyo valor pragmático es el mandato, el imperativo continúa siendo el recurso más eficaz desde el punto de vista comunicativo y, por consiguiente, el más habitual ${ }^{8}$. La preferencia por esta forma no es igual en todos los casos, sino que se observan situaciones y contextos más propicios y otros menos favorecedores. Aunque en ningún caso las diferencias son determinantes, sí son, al menos, interesantes.

En primer lugar, cabe destacar la repercusión que tiene la naturaleza semántica del verbo en la presencia del imperativo. De esta manera, vemos que los verbos de pensamiento, lengua o percepción aceptan en mayor proporción el imperativo que los verbos de acción o movimiento (93\% y 72\% respectivamente).

Asimismo, la relación que existe entre los interlocutores condiciona, en cierta medida, el uso del imperativo9. Se puede ver que cuando entre dos individuos existe una relación asimétrica del tipo [+ poder / - solidaridad], el porcentaje de imperativos aumenta sobre la media si el hablante posee el rasgo [+ poder] (89\%) y disminuye si su rasgo es [- poder] (69\%). Cuando la relación es de igualdad [- poder / + solidaridad] las cifras se sitúan entre las dos anteriores (69\%).

8 Solo tres de estos casos aparecieron junto a una forma de cortesía (por favor, si no te importa...), lo que permite calificar como extrañas en la conversación familiar este tipo de secuencias.

9 Esta variable está formada por tres variantes que se establecieron en función de las relaciones de poder y solidaridad que se daban entre los interlocutores. La variante a) implica que el informante X ejerce influencia sobre el informante $\mathrm{Y}$; en la variante b), es Y quien posee mayor poder; y en c), la relación es de solidaridad. El poder y la solidaridad se han determinado a partir de la edad y del parentesco, pues consideramos que en una red social como la alcalaína, constituida por familiares y amigos, eran los únicos factores que podían discriminar. Sobre los conceptos de poder y solidaridad véase R. Brown y A. Gilman, (1960). 
El factor sexo revela que las mujeres son más propensas al uso del imperativo que los hombres ( $85 \%$ frente a $67 \%$ ), lo que contradice la creencia popular de que el habla masculina es más "directa y ruda" que la femenina. Es muy posible que esta opinión se apoye exclusivamente en el léxico. Un análisis exhaustivo de unidades de mayor entidad formal desvelaría, tal vez, una realidad lingüística muy distinta.

Los datos en función de la edad indican que son los ancianos los más propensos a la forma imperativa, y que tal propensión disminuye según descendemos de generación; las diferencias entre la primera y la cuarta generación son muy significativas, y evidencian que se está produciendo un cambio en la concepción de la cortesía o, al menos, que la noción de cortesía (y por ende, el estilo de habla) varía según la edad del hablante.

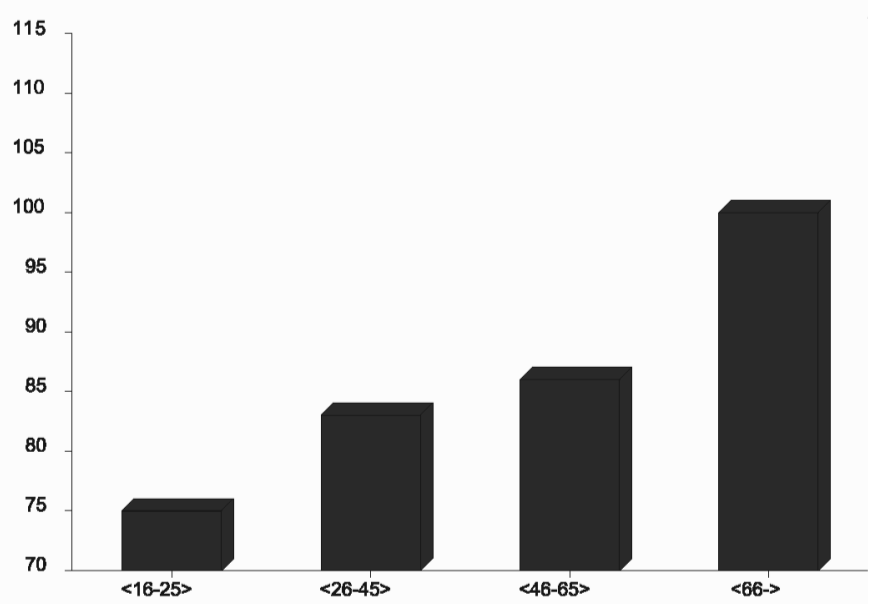

Gráfica 3: distribución del imperativo según la edad

En cuanto al nivel cultural, se aprecia igualmente una relación proporcional entre la presencia del imperativo y el nivel de formación, aunque inversa, es decir, a mayor grado de instrucción, menor porcentaje de este recurso. Sin embargo, las distancias entre variantes son, en esta ocasión, más modestas (superior $72 \%$, medio $73 \%$, inferior $87 \%$ ).

En muchas ocasiones aparece un tipo de imperativo, al que hemos denominado imperativo-fórmula, que no ha sido considerado en el estudio de la orden, ya que sus valores son otros. Contabilizamos 126 ejemplos (31\% del total de imperativos), con funciones y usos muy diversos:

- Causar sorpresa, llamar la atención sobre un hecho: Pues he conocido mi pueblo, fijate, pues hace, pues hace, 10 años que fui a mi pueblo. (inf. 2) 
- Dar una explicación, una justificación, un razonamiento: Hace veinte años o así ya empezó Alcalá a crecer porque mira, más o menos fue cuando ya se empezaron a hacer estos pisos. (inf. 2)

- Expresión de lo superlativo: Pues hablan malísimamente, con cada patada que le pegan al diccionario que, que no veas, ¿no? (inf. 2); Pero eso estaba ya, mira. (inf. 17)

- Cambiar de tema: Porque yo ahora mismo, mira mis nietos. (inf. 14)

- Mostrar desacuerdo: No creas, no pienses.

- Mostrar desconocimiento: Vete tú a saber.

- Coger el turno de palabra; este es uno de los valores más importantes: Mira, se ha levantado, se ha estado arreglando los cajones de su habitación (inf. 13). Mira, la cama se la hace porque la tiene que subir para arriba. (inf. 17)

\section{Otros recursos para la expresión de la orden y el mandato}

El presente de indicativo es, de este conjunto, el recurso más frecuente. Hay que destacar que con valor de orden y mandato se utiliza casi exclusivamente con verbos de acción y movimiento. Además, es mucho más frecuente en situaciones en las que los interlocutores mantienen una relación asimétrica del tipo b) ([- poder]). Por otra parte, es más común en los hombres, en especial, en los de la primera generación.

La incidencia del futuro en nuestros materiales es insignificante. Contrasta este hecho con la importancia que se concede a este valor en las gramáticas del español, lo que da una idea equivocada de la realidad lingüística, al menos, de la variedad centro-peninsular.

La estructura que + subjuntivo se utiliza normalmente para expresar mandato en aquellos casos en que no es posible el imperativo, esto es, para la primera persona del plural y la tercera singular y plural, bien sea porque el enunciado se dirija hacia estos sujetos, bien porque el carácter semántico del verbo no permita sujetos-agentes:

(5) No, no jugamos, pero que vaya a mirarlo. (inf. 5)

(6) Elein que vaya por su cuenta. (inf. 9)

(7) Que conste que no nos estáis dejando elegir. (inf. 9)

(8) Que se jodan. (inf. 12)

También encontramos estas secuencias en las transformaciones a estilo indirecto de órdenes directas (Rafa me ha dicho que si no venía más o menos pronto, que empezáramos a jugar - inf. 21), pero el hecho de ser discurso referido, transmisión de información, hace que la orden quede relegada a un segundo plano, es decir, el enunciado en sí no es una orden, es la expresión de una información 
que lleva implícita una orden. Por esta razón, no las hemos considerado para la cuantificación.

Las oraciones interrogativas son las formas más sutiles y corteses de ordenar; en ocasiones se presta a equívocos, dado ese carácter indirecto de expresar el contenido. Es por ello por lo que su frecuencia es muy baja en nuestros materiales. Piénsese que el análisis se ha hecho con un grupo de individuos que mantienen estrechas relaciones: la cortesía, en estos casos, no suele expresarse a través de elementos lingüísticos convencionales, sino que se recurre a otras formas como la gestualidad, la entonación, etc. Por otra parte, que la comunicación sea clara, eficaz y directa es lo primordial en los contextos comunicativos que hemos analizado.

\section{Conclusiones}

Los estudios de variación sintáctica deben superar las estrecheces de los planteamientos más puramente variacionistas (aplicados, sobre todo, a la fonética) y enfocarse desde una perspectiva más integradora y flexible, que dé cabida a cuestiones semánticas, pragmáticas y discursivas, esto es, deben desarrollarse dentro de una concepción funcional del lenguaje, pues, como señala M. J. Serrano (1999: 12), la variación "siempre es funcional, pues la producción lingüística es intención, estrategia y reflexión; está condicionada y condiciona la interpretación del locutor". Sobre estas bases, hemos analizado diferentes recursos con los que se expresan en español las modalidades epistémica, por un lado, y deóntica, por otro. Algunos de estos recursos no son, de ninguna manera, comparables funcionalmente, pero otros se utilizan, en contextos concretos, con la misma intención y son interpretados por el interlocutor de la misma manera. En ellos nos hemos centrado para nuestro estudio. Para el primer caso (expresión de la probabilidad y la posibilidad), hemos analizado cuatro recursos, dos de los cuales (futuro/condicional e indicativo + partícula) manifiestan notable vitalidad, mientras que los otros dos (subjuntivo + partícula y futuro/condicional + partícula) muestran un uso muy reducido. Hemos comprobado que la naturaleza semántica del verbo condiciona la mayor o menor presencia de determinados recursos; asimismo, los factores edad y nivel de instrucción inciden de alguna manera, y revelan que a menor edad y mayor nivel de instrucción (factores que suelen darse juntos en nuestros materiales), más variedad y riqueza expresiva. Por lo que respecta a la expresión de la orden y del mandato, uno de los recursos analizados -el imperativo- se ha mostrado claramente dominante. No obstante, hemos podido apreciar con claridad que existe correlación entre su aparición y los condicionantes bajo examen, poniendo de relieve un comportamiento interesante. Así, favorecen este recurso los verbos de pensamiento, lengua y percepción, las relaciones 
asimétricas de poder, las mujeres y los hablantes de mayor edad. Las diferencias son suficientemente significativas como para que nos plantemos la existencia de diversos sistemas de cortesía, que se darían en función de la edad, el sexo y la relación entre los hablantes.

\section{Referencias bibliográficas}

Aguado, J. A. (1987): "Uso discursivo de 'ir' en el habla culta de Sevilla". En Rodríguez Izquierdo, F. (ed.): Sociolingüística andaluza, 3. El discurso sociolingüístico. Sevilla, Universidad, págs. 121-136.

Alcaide, E. R. (1990): "La oración de predicado no verbal como medio de cohesión textual. Estudio contrastivo en las encuestas del Habla Urbana de Sevilla (niveles popular y culto)". En Palet Plaja, T. (ed.): Sociolingüistica andaluza, 5. Habla de Sevilla y hablas americanas. Sevilla, Universidad, págs. 125-136.

Bentivoglio, P. (1988): "La posición del sujeto en el español de Caracas: un análisis de los factores lingüísticos y extralingüístico". En Hammond, R. y M. C. Resnick (eds.): Studies in Caribbean Spanish dialectology. Washington, D.C., Georgetown University Press, págs. 13-23.

Bentivoglio, P. (1989): "Función y significado de la posposición del sujeto nominal en el español hablado". En Estudios sobre el español de América y lingüistica afroamericana. Bogotá, Instituto Caro y Cuervo, págs. 40-57.

Bentivoglio, P. (2003): "Orden de palabras en español: un análisis sintáctico-semántico-pragmático del sujeto", Lexis, 27/1-2, págs. 235-260.

Bolinger, D. (1977): Meaning and Form. London, Longman.

Cortés Rodríguez, L. (1986): Sintaxis del coloquio. Aproximación sociolingüística. Salamanca, Universidad.

Fuentes Rodríguez, C. (ed.) (1993): Sociolingüistica andaluza, 8. Estudios sobre el relato oral. Sevilla, Universidad.

Guillén, R. (1990): "Uso discursivo de CREER y PENSAR en el habla urbana de Sevilla". En Palet Plaja, T. (ed.): Sociolingüistica andaluza, 5. Habla de Sevilla y hablas americanas. Sevilla, Universidad, págs. 85-102.

Jacobson, S. (1989): "Some Approaches to Syntactic Variation". En Falsod, R. y D. Schiffrin (eds.): Language Variation and Change. Washington, Georgetown University Press, págs. 381-394.

Jiménez Juliá, T. (1989): "Modalidad, modo verbal y modus clausal en español", Verba 16, págs. 175-214.

Kitova-Vasileva, M. (2000): La verosimilitud relativa su expresión en español. Santiago de Compostela, Servicio de Publicaciones de la Universidad de Santiago de Compostela.

Labov, W. (1996): The social stratification of English in New York City. Washington, Center for Applied Linguistics.

Lavandera, B. (1984): Variación y significado. Buenos Aires, Hachette. 
Lorenzo, E. (1971): "La expresión del ruego y del mandato". En El español de hoy. Lengua en ebullición. Madrid, Gredos, págs. 84-96.

Lyons, J. (1980): Semántica. Traducción de R. Cerdá, Barcelona, Teide.

Maingueneau, D. (1980): "Las modalidades". En Introducción a los métodos de análisis del discurso. Buenos Aires, Hachette, págs. 128-130.

Martín Butragueño, P. (1994): "Hacia una tipología de la variación gramatical en sociolingüística del español”, Nueva Revista de Filología Hispánica, XLII, págs. 28-75.

Matte Bon, F. (1995): Gramática Comunicativa del español II. De la idea a la lengua. Madrid, Edelsa.

Medina López, J. (1993): Formas de tratamiento en una comunidad rural (Buenavista del Norte. Tenerife). Santa Cruz de Tenerife, Ilmo. Ayto. de Buenavista del Norte/Viceconsejería de Cultura del Gobierno de Canarias.

Meunier, A. (1974): "Modalités et communication”, LFr 21, págs. 23-46.

Moreno Fernández, F. (1989): "Análisis sociolingüístico de actos de habla coloquiales (I)", EA, 51, págs. 5-51 y "Análisis sociolingüístico de actos de habla coloquiales (II), EA, 52, págs. 5-57.

Nuñez, S. (1991): Semántica de la modalidad en latín. Granada, Servicio de Publicaciones de la Universidad de Granada.

Otaola Olano, C. (1988): "La modalidad (con especial referencia a la lengua española)", Revista de Filología Española, Tomo LXVIII, fascículo $1^{\circ}$ - 2º págs. 98-101.

R. Brown, R. y Gilman, A. (1960): "The pronoums of power and solidarity". En Sebeok, T. (ed.): Style in language. New York, John Wiley, 253-276.

Real Academia Española. (1978): Esbozo de una Nueva Gramática de la Lengua Española. Madrid, Espasa-Calpe.

Ridruejo, E. (1999): "Modo y modalidad. El modo en las subordinadas sustantivas". En Bosque, J. I. y V. Demonte (dirs.): Gramática descriptiva de la lengua española. añola. Madrid, Espasa-Calpe, págs. 3209-3251.

Rodríguez-Izquierdo, F. (1987): "Procedimientos de negación en el habla de Sevilla" y "Procedimientos de topicalización en el habla culta de Sevilla". En Rodríguez Izquierdo, F. (ed.): Sociolingüística andaluza, 3. El discurso sociolingüístico. Sevilla, Universidad, págs. 137-154 y 31-49.

Romaine, S. (1984): "On the Problem of Syntactic Variation and Pragmatic Meaning in Sociolinguistic Theory”, Folia Lingüística 18, págs. 409-437.

Sánchez Lanza, C. (1988): "Formas de mandato y ruego en la lengua oral de Rosario". En Actas del IV Congreso Internacional de ALFAL, México, UNAM, págs. 849-862.

Sankoff, D. (1992): "Sociolingüística y variación sintáctica". En Newmeyer, F. J. (ed.): Panorama de la lingüística moderna de la Universidad de Cambridge, IV. El lenguaje: contexto sociocultural. Madrid, Visor, págs. 173-196.

Serrano, M. J. (1994): La variación sintáctica: formas verbales del período hipotético en español. Madrid, Entinema.

Serrano, M. J. (ed.) (1999): Estudios deEstudios de variación sintáctica. Madrid, Iberoamericana. 
Silva-Corvalán, C. (1981): "The difusion of object-verb agreement in Spanish", Papers in Romance, 3, págs. 163-176.

Silva-Corvalán, C. (1982): "Subjetc variation in spoken Mexican-American Spanish". En Spanish in the United States: Sociolinguistics aspects. New York, Cambridge University Press, págs. 93-120.

Silva-Corvalán, C. (1984): "Topicalización y pragmática en español”, Revista Española de Lingüística, 14, págs. 1-19.

Silva-Corvalán, C. (1980-81): "La función pragmática de la duplicación de pronombres clíticos”, Boletín de Filología de la Universidad de Chile, XXXI, págs. 561-570.

Sversidad de Chile, XXXI, págs. 561-570.

Silva-Corvalán, C. (1988): Sociolingüística. Teoría y análisis. Madrid, Alhambra.

Silva-Corvalán, C. (2001): Sociolingüística y pragmática del español. Washington, D. C., Georgetown University Press. 